sehr genau die bisher vorhandenen Versicherungsformen und ihre Leistungsfähigkeit dar, nachdem der Verfasser einen kurzen Einblick (S. 29-59) in die soziale Lage der Bevölkerung gegeben hat. Die Ausgangsthese, die sicher richtig ist, ist die, daß der indische Arbeiter keine Einkommensüberschüsse hat, die er für die Sozialversicherung benutzen kann (S. 57). Auch die dörfliche Familie, aus der viele Arbeiter kommen, kann hier nicht helfen, weil sie sogar umgekehrt auf die Hilfe der in die Stadt gegangenen Familienmitglieder angewiesen ist (S. 59). Von daher kommt von vornherein nur ein Pflichtversicherungssystem in Betracht. Mit dem „Employees' State Insurance Act 1948“ wurde eine Pflichtversicherung für Industriearbeiter mit einem Monatseinkommen bis 400 Rps eingeführt. Die Versicherung sollte in erster Linie den Bedürftigsten zugute kommen. Dies Argument mag für die erste Zeit, als die Versicherung noch auf große Staatszuschüsse angewiesen war, gelten. Inzwischen gilt es nicht mehr und sollte schleunigst aufgegeben werden. Vielmehr zeigen die Relationen von Ausgaben und Einnahmen, je höher das Einkommen ist, desto größere Überschüsse (vgl. Tabelle S. 77). Eine Anhebung der Pflichtversicherungsgrenze wäre also dringend zu empfehlen (S. 198)!

Hauptnachteil des Systems ist die enge sachliche Begrenzung. Zwar waren bereits 1966 3,4 Mio versichert, aber die große Masse der Bevölkerung, die auf dem Lande lebt, bleibt ebenso ausgeschlossen wie die Bereiche Handel und Kleingewerbe, da nur Betriebe mit mindestens 10 Arbeitnehmern erfaßt werden. Solche Ausdehnung läßt sich natürlich nicht von heute auf morgen schaffen, aber die beträchtlichen Anfangserfolge der Industriearbeiter-Versicherung, die allerdings in Sach- und Geldleistungen nach wie vor ungenügend ist, lassen eine allgemeine Sozialversicherung doch möglich erscheinen.

Das Buch Heinemanns ist außerordentlich zu begrüßen, weil es ein ganz wesentliches Element der Sozialpolitik an Indien exemplarisch darstellt. Dies ist um so mehr zu begrüßen, als es selbst in Indien eine wirkliche Gesamtdarstellung nicht gab. Schade, daß das Buch nicht auf englisch erschienen ist, dann hätte es anderen Entwicklungsländern noch besser zum Vergleich und als Modell dienen können.

Henning v. Wedel

\title{
Detlef Kantowsky
}

Dorfentwicklung und Dorfdemokratie in Indien

Freiburger Studien zu Politik und Gesellschaft überseeischer Länder Band 9 Bertelsmann Universitätsverlag, Bielefeld 1970, 171 S.

Henry Maddick

Panchayati Raj

Inlogov (International Local Government) Longman, London 1970, XIV, 402 S., $£ 4.50$

\section{KIEMENS VAN DE SAND}

Grundlagen und Probleme der ländlichen Lokalverwaltung in Indien

Mitteilungen des Instituts für Asienkunde Nr. 54, Hamburg 1973, 202 S.

Eines der großen Probleme der Entwicklungsländer ist die Entwicklung der Landwirtschaft. Dies wird nicht nur durch die Untersuchung des Entwicklungsprozesses der Industrienationen nahegelegt, bei denen ein Zusammenhang zwischen Leistungs- 
fähigkeit der Landwirtschaft und Entwicklungspotential nachgewiesen ist. Vor allem fordert die rasante Bevölkerungszunahme ein effizientes produktives Landwirtschaftssystem. Zugleich verlangt der Aufbau eines modernen Staates auch eine Erfassung der ländlichen Gebiete, was um so mehr gilt, als in fast allen Entwicklungsländern der größte Teil der Bevölkerung auf dem Lande lebt. Communitydevelopment, Dorfentwicklung, ist deshalb eines der im Brennpunkt des Interesses stehenden Entwicklungsprobleme.

Die angezeigten Bücher befassen sich mit diesen Programmen in Indien, das in vielerlei Hinsicht als Prototyp des Entwicklungslandes gelten kann. Die Bevölkerung hat sich in den letzten 40 Jahren mehr als verdoppelt. 80 Prozent der Bevölkerung leben auf dem Lande. Die Landwirtschaft ist zum größten Teil reine Subsistenzwirtschaft, der Grundbesitz im Durchschnitt gering. Erst in neuester Zeit gehen die Großgrundbesitzer zur Eigenbewirtschaftung über, während früher die Unterverpachtung in Kleinparzellen bei 50prozentiger Erntebeteiligung die Regel war.

Das Buch von K. van de Sand gibt vor allem eine historische Übersicht über Wurzeln und Entstehung des indischen Dorfentwicklungsprogramms, das schließlich im System der Panchayati Raj seine endgültige Form gefunden hat. Der Begriff Panchayat, der wörtlich „Rat der Fünf“ heißt (S. 13) und heute als Verwaltung oder Herrschaft durch selbstgewählte Räte adäquat übersetzt werden müßte, ist sehr alt und bezeichnet das Verwaltungssystem im traditionellen Indien, wo jedes Dorf weitgehend selbständig und mit der Oberhoheit des Monarchen nur durch die Steuerpflicht verbunden war. Jedoch bestand damals kein einheitliches System, vielmehr gab es Räte der verschiedensten Zusammensetzung mit auch ganz verschiedenen Aufgaben unter oder neben anderen Formen der dörflichen Verwaltung (S. 20). Die Dorfgemeinschaft trat als ganze dem Steuereinnehmer gegenüber und war damit eigener Träger von Verwaltungsfunktionen (S. $21 \mathrm{ff}$.).

Mit der Entwicklung eines modernen Steuer- und Verwaltungssystems wurde der dörflichen Selbstverwaltung der Boden entzogen (S. 36, 58). An deren Stelle trat eine Distriktsverwaltung, die nach und nach den entscheidenden Anteil an der Verwaltung Britisch-Indiens überhaupt an sich zog. Der damit verbundene Verlust von Initiative und Mitwirkungsmöglichkeiten der Landbevölkerung führte bereits Anfang des 19. Jahrhunderts zu Reformbestrebungen mit dem Ziel, die dörfliche Selbstverwaltung wieder aufzubauen. Dabei wurde anknüpfend an historische Vorbilder (S. 14 ff.) der Panchayat als Schlichtungs- und Gerichtsinstanz für Bagatellsachen wieder eingeführt (S. 61), allerdings ohne großen Erfolg. Auch die große von Lord Rippon am Ende des 19. Jahrhunderts eingeleitete Reform blieb letztlich erfolglos (S. 69), hat aber deshalb ungeheure Bedeutung, weil sie das Idealbild einer dörflichen Selbstverwaltung am Leben erhielt und so die Grundlage auch für die heutige Form des Selbstverwaltungssystems bildet. Weitere Reformvorschläge und Gesetzesvorhaben folgten. Letztlich aber blieb es bei der Distriktsverwaltung, der Collector als Distriktschef blieb alleinverantwortlich. Steuereintreibung und Aufrechterhaltung der Ordnung durch eine dekonzentrierte (nicht dezentralisierte) Verwaltung standen im Vordergrund. Der Aufbau echter Selbstverwaltung und die Beteiligung der breiten Bevölkerung an der Verwaltung gelangen nicht (S. 82).

Im unabhängigen Indien wurden mit der Selbstverwaltung die landwirtschaftlichen Entwicklungsprogramme verknüpft. Die Selbstverwaltungsorgane sollten zugleich 
die Entwicklungsplanung, aber auch die Planausfüllung und Finanzierung übernehmen. Neue wichtige Verwaltungseinheit wurde der "Development Block“, der die Arbeit der Dorfentwicklungsräte koordinieren sollte. Diese Mittelstufe im dreistufigen Aufbau: Dorf, Block, Distrikt, ist durch die Panchayat Samitis in das jetzige System übernommen worden (S. 97). Daneben bestand aber die alte Distriktsverwaltung weiter, worauf van de Sand auch das Scheitern dieses ersten Community Development Program zurückführt (S. 91 f.). Im folgenden Abschnitt stellt der Verfasser das zwischen 1959 und 1962 eingeführte „Panchayati Raj System" dar (S. 94-139). In einem abschließenden Teil (S. 141-180) untersucht dann der Verfasser die Auswirkungen der historischen Entwicklung auf das heutige System und die sich daraus ergebenden Probleme.

Die Gründe für das weitgehende Scheitern der früheren Programme und die Probleme des heutigen Systems sieht van de Sand vor allem in der fehlenden Relevanz der dörflichen Gemeinschaft für das Leben des einzelnen. Kaste, Familie und hierarchische Verwaltung lassen dem so hierarchisch Eingeordneten keine Freiheit für Selbstverwaltung auf der Ebene einer prinzipiellen Gemeinschaft. Dieses Problem hat sich nach Meinung des Verfassers noch verstärkt durch die Planwirtschaft und die damit notwendig verbundene Zentralisierung (S. $181 \mathrm{ff}$.). Selbstverwaltung, wie alle Demokratisierung, setzt zunächst einmal prinzipielle Gleichheit voraus. Das "Panchayati Raj" hat diese Gleichheit nicht geschaffen und konnte das auch nicht. Der Verfasser glaubt aber, daß das System zur Politisierung der Bevölkerung beitrage. So bleibt zu hoffen, daß eines Tages ein neues Bewußtsein die alten hierarchischen Bindungen und die ebenso strukturierte Verwaltung aufbrechen und wirkliche Selbstverwaltung ermöglichen wird.

Legt das Buch von van de Sand den entscheidenden Akzent auf die Historie, so stellt Maddick in erster Linie das geltende System dar. Nach einer Einleitung, die etwas geraffter und mit etwas weniger Rücksicht auf die Beziehungen zum Steuersystem in die heutige Situation einführt (S. 3-64), stellt Maddick ausführlich das System der Panchayati Raj dar. Er hat (wie übrigens auch van de Sand, der nur weniger spezifisch) die Staaten Maharashtra, Rajasthan und Andhra Pradesh in den Mittelpunkt der Beobachtung gestellt, gibt aber auch die wesentlichen Züge des Systems in den anderen Staaten an. Die Vielfalt der Systeme rührt von der Zuständigkeit der Staaten für die Gesetzgebung über die Selbstverwaltung her (Parallelen zur deutschen Gemeindeverwaltung drängen sich auf). Maddick gibt eine klare Darstellung, die durch Übersichtstabellen noch leichter zugänglich und für Vergleiche nutzbar wird. Die Fülle des Materials und die Schwierigkeit zu generalisieren hebt Maddick unter Hinweis auf die Größe, die Staaten- und Sprachvielfalt und die ungeheure Bevölkerungszahl hervor (S. 306). Gleichwohl gibt er einige interessante zusammenfassende Hinweise (S. $307 \mathrm{ff}$.).

Insgesamt beurteilt er die Grundlage und das System als solches als brauchbar. Jedoch seien die Beziehungen zwischen den Selbstverwaltungsorganen und den Regierungen nicht so gut, wie sie es sein sollten. Die Personalpolitik variiere stark und reiche von unüberlegt bis vorsichtig vernünftig. Die finanzielle Ausstattung sei allgemein ungenügend. Die Attitüde der Politiker gegenüber der Selbstverwaltung sei persönlich sehr unterschiedlich. Von Ablehnung über Uninteressiertheit bis zur Ausnutzung für die eigene Karriere ist alles vertreten. Die Parteien insgesamt dagegen hätten das System angenommen. Da es aber an genauerer Forschung fehle, seien in diesem Bereich noch weniger als sonst Urteile möglich, da sie sich 
nur auf Gefühle und zufällige Information stützen könnten. Die Zukunft der indischen Politik liegt nach Maddick auf dem Lande und je mehr Zuständigkeiten nach dort abgegeben wurden, desto mehr wird das Selbstverwaltungssystem zur Effizienz gezwungen, desto mehr Chancen bieten sich aber auch für einen soliden politischen Aufbau von unten nach oben. Die Räte geben die Chance auch bisher ausgeschlossenen Kasten und Klassen, sich am politischen Geschehen zu beteiligen, wobei natürlich die jeweilige Struktur (in Maharashtra besonders fortschrittlich) hemmend oder förderlich wirkt. (Hier decken sich Maddicks und van de Sands Urteile.) Auch Maddick sieht aber die Gefahr, daß das traditionell hierarchisch gegliederte politische System die Selbstverwaltung nicht zur Entfaltung kommen läßt, zumal es ohnehin schwer ist, für ein so breites Programm ausreichend qualifizierte Leute sowohl auf der Beamten- wie aber noch mehr in der Ratsebene zu finden. Wegen der Unfähigkeit zahlreicher gewählter Dorfvertreter hat es denn auch zu Protesten und erneuten Reformansätzen, ja sogar zum Ruf nach Abschaffung der gesamten Selbstverwaltung geführt. So problematisch die Verbindung von Selbstverwaltung und Entwicklungsprogrammen ist, so glaubt Maddick doch, die generelle Fähigkeit zur Entwicklung durch das Selbstverwaltungssystem konstatieren zu können. Er sieht in der staatlichen Planung keine echte Gefahr, weil diese doch nur durchgeführt und effektiv gemacht werden kann, wenn die Gemeinden und Dörfer die Pläne annehmen und adäquat umsetzen.

Das Endurteil schließlich ist abgewogen, das System ist kein Deus ex machina für Demokratisierung, aber es ist ein wesentlicher, aus der indischen Gesellschaft nicht mehr wegzudenkender Faktor auf dem Wege der Demokratisierung eines in jahrhundertelanger autoritärer Herrschaft in politischer Apathie versunkenen Volkes.

Vergleicht man die beiden Bücher, so muß man unbedingt das Buch von Maddick empfehlen. Es ist breiter angelegt, materialreicher und unvergleichlich genauer als das Buch von van de Sand. Die etwas umfangreichere historische Darstellung van de Sands gibt keinen echten Gewinn. Die aus der Historie abgeleiteten Schlüsse über die Tauglichkeit des heutigen Systems sind weitgehend die gleichen wie die aus der Analyse des Vorhandenen gefundenen. Nur wer mit der englischen Sprache Schwierigkeiten hat, sich aber trotzdem einen Einblick verschaffen will, mag zu dem Buch von van de Sand greifen. Ansonsten empfiehlt es sich, das gewissermaßen ein Handbuch darstellende Werk Maddicks vorzuziehen, obwohl dieser sein Material weitgehend vor 1966 gesammelt hat. Auch der spätere Zeitpunkt der Materialsammlung bei van de Sand (1966-1971) rechtfertigt kein anderes Urteil, da beide Bücher sich mehr mit den Grundlagen als der aktuellen Entwicklung befassen, zumal van de Sand neueres Tatsachenmaterial wegen seines historischen Ansatzes kaum verwertet.

Mit den direkten Auswirkungen der Dorfentwicklungsprogramme beschäftigt sich die Konstanzer Habilitationsschrift D. Kantowskys. Er hat in drei Dörfern in Uttar Pradesh die tatsächlichen Funktionen und Erfolge auf der Bezugsebene des ganzen Programms, nämlich dem Dorf, untersucht und dabei zugleich einen wesentlichen Beitrag zum Verständnis des modernen ländlichen Indien geleistet. Da Kantowsky sich auf einen Block und drei Dörfer beschränkt, sind seine Aussagen zwar nicht allgemein, dafür aber um so eindeutiger und genauer. Das Buch Kantowskys zeigt dem Leser die bei den anderen Büchern nur vermuteten tatsächlichen Schwierigkeiten. Hier treten Probleme nicht als abstrakte Dysfunktionalitäten, sondern als persönliche und unmittelbare sachliche Fehlschläge oder Lösungsschwierigkeiten 
auf. Kantowsky hebt vor allem die Kaste als Mittel der sozialen Einordnung hervor und weist nach, daß nach wie vor Zugehörigkeit zu einer der oberen Kasten und Landbesitz die entscheidenden Kriterien bei der Verteilung der knappen Resourcen und der Posten sind. Das Selbstverwaltungssystem dient nach Kantowsky keineswegs dazu, die sozialen Spannungen abzubauen, wie es Ghandi vorschwebte, sondern es verstärkt sie sogar. Geht man davon aus, daß politische Systeme soziale Konflikte meistern oder sogar aufheben sollen, so ist „Pnachayati Raj“ dafür ungeeignet. Kantowskys Prognose ist durchaus pessimistisch. Er glaubt nicht, daß das System langfristig ein Erfolg werden kann. Selbst wer da anderer Ansicht ist, sollte das Buch Kantowskys zur Kenntnis nehmen. Er versucht eine Gesamtanalyse der Bestimmungen, der faktischen Organisation und der tatsächlichen Auswirkungen im Dorf. Jeder, der sich für Indien speziell aber auch jeder, der sich für Entwicklungsplanung und Entwicklungshilfe generell interessiert, sollte dieses Buch lesen, um zu erkennen, wie viele Probleme, wieviel menschliches Leid und wieviel soziale Dynamik hinter den sonst überall allein mitgeteilten Zahlen und abstrakten Programmen stehen. Obwohl auch dieses Buch sehr viel Zahlenmaterial, Tabellen und Diagramme enthält, bietet es einen gut lesbaren unmittelbaren Einblick in die dem Programm zugrundeliegende soziale Wirklichkeit und die Auswirkungen des Programms in ihr.

Die beiden Bücher von Maddick und Kantowsky ergänzen sich ideal. Zusammen geben sie einen wirklich guten, fundierten Überblick über das indische Dorfentwicklungsprogramm, das immer stärker zum Modellfall für andere Länder avanciert.

Henning v. Wedel

\section{KARL-Dieter OpP}

\section{Soziologie im Recht}

rororo. Studium, Reinbek 1973, 265 S., 9,80 DM

Die Erforschung des Rechts der Entwicklungsländer erfordert die Fähigkeit, Recht in seinen Beziehungen zur sozialen Wirklichkeit zu sehen. Für diese Aufgabe sind deutsche Juristen von ihrer Ausbildung her nur unzureichend vorbereitet. Es ist daher erfreulich, daß die Diskussion über die Notwendigkeit einer stärkeren Berücksichtigung der Sozialwissenschaften in der juristischen Ausbildung und Praxis auch ein rasches Anwachsen rechtssoziologischer Literatur zur Folge hat. Aus dieser hebt sich das anzuzeigende Buch in dreierlei Hinsicht heraus:

1. es ist für den „Durchschnittsjuristen " sprachlich zugänglich ${ }^{1}$;

2. es ist kooperationsbereit geschrieben, d. h. nicht im Stil der Arbeiten die Schelsky polemisch als "Diffamierungssoziologie von Recht und Juristen“ bezeichnet hat ${ }^{2}$, und die - ganz unabhängig von ihrem Wahrheitsgehalt - bei Juristen eher Verteidigungsmechanismen als Interesse an Sozialwissenschaften auslösen;

3. es legt relativ konkrete Ergebnisse vor, und nicht nur allgemein gehaltene $\mathrm{Be}-$ merkungen über zukünftige Forschungsprogramme und Kooperationsmöglichkeiten.

\footnotetext{
1 Während ein Rezensent vermutet, daß dieser Durchschnittsjurist z. B. Luhmanns „Rechtssoziologie* (Reinbek 1972) spätestens auf S. 35 aus der Hand legen wird (Rasehorn JZ. 1973, S. 17).
}

2 Jahrbuch für Rechtssoziologie und Rechtstheorie Bd. 3 (1972), S. 610. 